UCRL-JC-130030

Preprint

\title{
Modeling of Frequency Doubling and Tripling with Converter Refractive Index Spatial Non-Uniformities due to Gravitational Sag
}

\author{
J. M. Auerbach, C. E. Barker, S. A. Couture, D. Eimerl, J. J. De Yoreo, \\ L. A. Hackel, R. L. Hibbard, L. W. Liou, M. A. Norton, S. A. Perfect, P. J. Wegner
}

This paper was prepared for submittal to Third International Conference on Solid State Lasers Application (SSLA), Inertial Confinement Fusion(ICF) Monterey, CA June 7-12, 1998

August 3, 1998

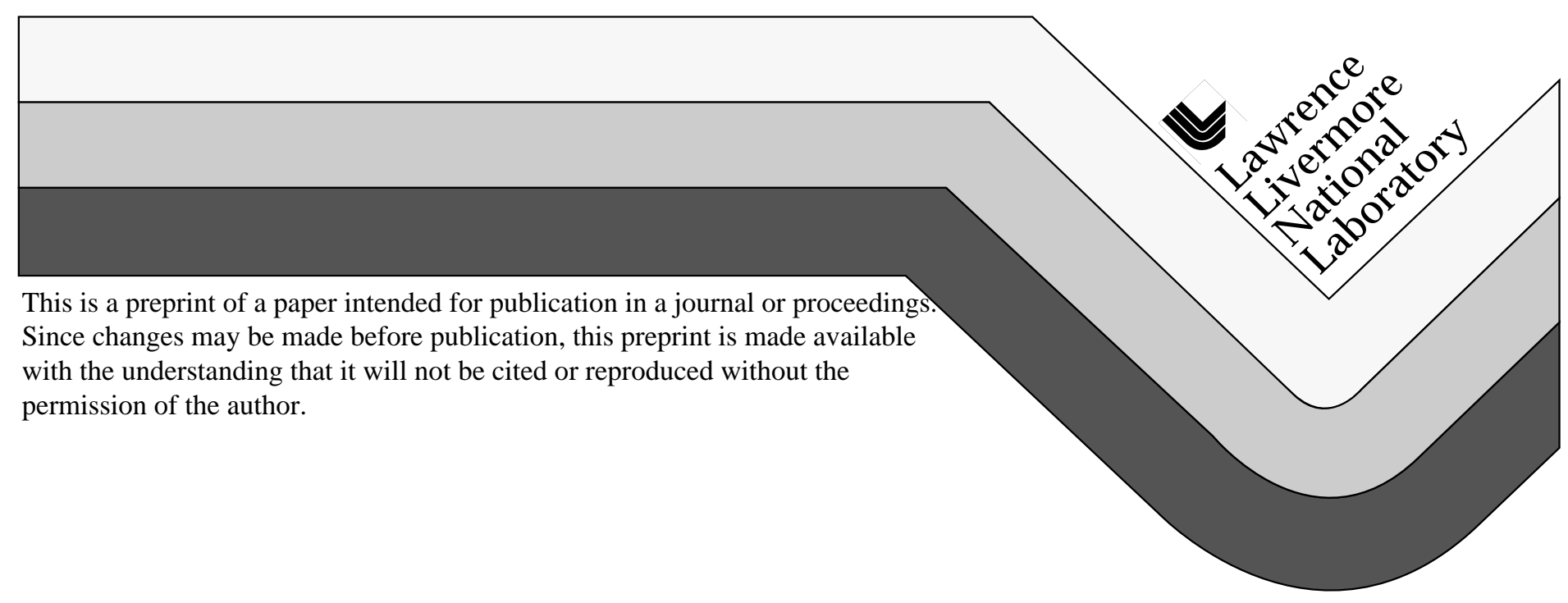




\section{DISCLAIMER}

This document was prepared as an account of work sponsored by an agency of the United States Government. Neither the United States Government nor the University of California nor any of their employees, makes any warranty, express or implied, or assumes any legal liability or responsibility for the accuracy, completeness, or usefulness of any information, apparatus, product, or process disclosed, or represents that its use would not infringe privately owned rights. Reference herein to any specific commercial product, process, or service by trade name, trademark, manufacturer, or otherwise, does not necessarily constitute or imply its endorsement, recommendation, or favoring by the United States Government or the University of California. The views and opinions of authors expressed herein do not necessarily state or reflect those of the United States Government or the University of California, and shall not be used for advertising or product endorsement purposes. 
*Modeling of Frequency Doubling and Tripling with Converter Refractive Index Spatial Non-Uniformities Due to Gravitational Sag

J.M. Auerbach, C.E. Barker, S.A. Couture, D. Eimerl, J.J. De Yoreo, L.A. Hackel, R.L. Hibbard, L.W. Liou, M.A. Norton, S.A. Perfect, P.J. Wegner

University of California, Lawrence Livermore National Laboratory, P.O. Box 808 Livermore, CA 94550

\begin{abstract}
Accurate predictions of the performance of frequency conversion requires knowledge of the spatial variation of departures from the phase-matching condition in the converter crystals. This variation is caused by processes such as crystal growth and crystal surface finishing. Gravitational sag and mounting configurations also lead to deformation and stresses which cause spatially varying departures from the phase-matching condition. We have modeled the effect of gravitational forces on conversion efficiency performance of horizontal converter crystals and have shown for the NIF mounting configurations that gravity has very little effect on conversion efficiency.
\end{abstract}

Keywords: Frequency conversion, ICF, Nonlinear optics, KDP crystals

*Work performed under the auspices of the U.S. Department of Energy by Lawrence Livermore National Laboratory under Contract No. W-7405-Eng-48. 


\section{BACKGROUND}

Efficient frequency doubling and tripling occurs at the phase matching condition. For Type I frequency doubling, the phase velocities of the second harmonic $(2 \omega)$ and fundamental $(1 \omega)$ waves are equal at the phase-matching condition. For Type II frequency tripling, the phase velocities of the third harmonic, second harmonic and fundamental waves are equal. In this paper, we describe the modeling of the effects of gravity forces on the phase-matching condition in Type I KDP doublers and the resultant effect on frequency tripling efficiency. We have neglected in this analysis the effects of gravity sag on the tripler crystal since it has much lower angular sensitivity than the doubler. For Type I doubling the phase mismatch factor $\Delta \mathrm{k}$ is given by:

$$
\Delta \mathrm{k}=4 \pi / \lambda\left[\mathrm{n}_{\mathrm{e}}\left(\theta, 2 \omega, \sigma_{\mathrm{ij}}\right)-\mathrm{n}_{\mathrm{o}}\left(\omega, \sigma_{\mathrm{ij}}\right)\right]
$$

where $\theta$ is the angle between the optical axis and the incoming ray, and $\sigma_{\mathrm{ij}}$ is the stress tensor; $\mathrm{n}_{\mathrm{o}}$ is the ordinary refractive index; and $\mathrm{n}_{\mathrm{e}}$ is the extraordinary refractive index. The modeling results in this paper will show the effect on $\Delta \mathrm{k}$ due to the crystal being subjected to gravity and different mounting configurations. The Type I doubler is the crystal in the NIF converter with the highest angular sensitivity. The anisotropic mechanical and optical properties of KDP were obtained from reference 1.

\section{MODELING OF EFFECTS OF GRAVITY ON FREQUENCY CONVERSION}

\subsection{Crystal configurations for the modeling}

We modeled the deformation and stress in the KDP Doubler using the Lawrence Livermore National Laboratory finite element code NIKE3D ${ }^{2}$. This code can utilize anisotropic material properties. The Type I KDP crystal plates used for the modeling had dimensions of $37 \mathrm{~cm} \times 37 \mathrm{~cm} x 1 \mathrm{~cm}$. A grid of 47x 47x7 node points was defined for the plate. Displacements were calculated for each node point. The stress tensor was calculated in elements bounded by each set of adjacent 8 node points.

Three mounting configurations were studied

(1) Supported at 4 corner

(2) Simply supported around full periphery

(3) Clamped around full periphery.

For case (3), one crystal surface has a linear clamping force applied to it through a compliant strip with an elastic modulus of $200 \mathrm{lbs} / \mathrm{in}^{2}$. For the modeling a linear clamping force $1.0 \mathrm{lb} / \mathrm{in}$ was used.

\subsection{Effects of gravity-induced deformation on doubler converter crystal performance}

Figure 1 shows a schematic of a crystal with gravity sag and an incoming ray. For elastic deformations, the phase matching direction is normal to the crystal surface. Finite element code calculations have also shown that the phase-matching direction for a given $\mathrm{x}$, y location is the same at all distances into the crystal. The angle between the initial ray direction and the surface normal divided by the refractive index is the local detuning angle. This local detuning angle is the parameter that relates a change in conversion efficiency to a deformation of the crystal surface due to gravity. The displacement or sag itself is not important for frequency conversion; the important quantity is the local slope of the surface. We have calculated the local detuning angle distributions for the three configurations listed above. Figure 2 shows the detuning angle distribution for the doubler crystal mounted at 4 corners. The four corner support configuration results in detuning angles that would result in very large drops in doubling efficiency so it is no longer considered for NIF. Figure 3 
shows the calculated detuning angle distribution for a Type I doubler simply supported(no clamping moments) around the full periphery. Figure 4 shows the detuning angle distribution for the Type I doubler clamped around the periphery with a compliant strip. The corresponding displacements are small for this clamped doubler. Figure 5 shows the calculated z-displacement distribution. The center of the plate sags down only 2.6 microns relative to the edges.

We modeled the effect of this doubler crystal deformation on tripling efficiency by applying the doubler detuning angle distribution to the NIF baseline converter which consists of:

$11 \mathrm{~m}$ thick KDP Type I doubler, detuning angle(internal)=240 $\mathrm{rad}$

\section{9mm thick KD*P Type II tripler}

All surface reflectivities were assigned a value of $0.5 \%$. The drive consisted of ideal $1 \omega$ plane waves with intensities of 3,4 , and $5 \mathrm{GW} / \mathrm{cm}^{2}$. Figure 6 shows the $3 \omega$ conversion efficiencies at each value of the detuning angle distribution $(240 \mu \mathrm{rad}+$ each internal angle in figure 4$)$. The plots show very little variation in the conversion efficiency over the detuning angle range. Based on a histogram of the detuning angle distribution, the overall $3 \omega$ conversion efficiency was calculated for the three $1 \omega$ intensities. The values are:

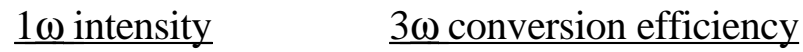

$\begin{array}{ll}3.0 \mathrm{GW} / \mathrm{cm}^{2} & 87.23 \% \\ 4.0 \mathrm{GW} / \mathrm{cm}^{2} & 90.51 \% \\ 5.0 \mathrm{GW} / \mathrm{cm}^{2} & 92.17 \%\end{array}$

From figure 6 and these values it can be concluded that for the doubler in a clamped mounting configuration, the effect of gravity-induced deformation on tripling efficiency is very small.

\subsection{The Stress-Optic effect}

In addition to gravity deforming the crystal and causing local detuning angles, it also leads to a stress distribution in the crystal which alters the phase-matching condition. This occurs through the stress-optic effect ${ }^{3,4}$ in which the reflective index at a point in a material is altered by the stress at that point.

Pockels' stress-optic threory relates changes in refractive index to stress. Let $\mathrm{B}_{\mathrm{ij}}$ denote the impermeability tensor. $\mathrm{B}_{\mathrm{ij}}$ is related to the electric displacement tensor $\mathrm{D}_{\mathrm{ij}}$.

$$
\begin{aligned}
D_{i j} & =\varepsilon K_{i j} \\
B_{i j} & =1 / K_{i j}
\end{aligned}
$$

The eigenvalues of $\mathrm{B}_{\mathrm{ij}}$ are $1 / \mathrm{n}^{2}{ }_{\mathrm{kk}}$ where $\mathrm{n}$ is the refractive index for direction $\mathrm{k}$. Pockels' law relates a change in the impermeability tensor $\Delta \mathrm{B}_{\mathrm{ij}}$ to the stress tensor $\sigma_{\mathrm{ij}}$.

$$
\Delta \mathrm{B}_{\mathrm{ij}}=\pi_{\mathrm{ijkl}} \sigma_{\mathrm{kl}}
$$


$\pi_{\mathrm{ijkl}}$ is the piezo-optic coefficient tensor. Values of this tensor are available ${ }^{1}$ for KDP. Modeling of stress-optic effects in the KDP Type I doublers results in finding the value of $n_{e}(2 \omega)-n_{0}(\omega)$ at each element in the crystal plate grid. This can then be used to calculate the variation in conversion efficiency due to the stress distribution. We calculate these indices as follows. First NIKE3D is used to calculate the stress tensor at each element in the crystal. $\Delta \mathrm{B}_{\mathrm{ij}}$ is then calculated using equation (4) and these values are used to calculate the updated values of $B_{i j}$ for each element. Then the eigenvalues of the subset of $B_{i j}$ in the plane of the crystal are dimensions are calculated at wavlengths corresponding to $1 \omega$ and $2 \omega$. Knowing that these eigenvalues correspond to $1 / n^{2}, n_{o}(1 \omega)$ and $n_{e}(2 \omega)$ are readily calculated.

Using NIKE3D, we calculated the stress tensor for each element of a horizontal KDP Type I doubler plate subjected to gravity and clamped around its full periphery with a clamping force of 1.0 $\mathrm{lb} / \mathrm{in}$. The clamping was done through a compliant strip which has an elastic modulus of $200 \mathrm{lbs} / \mathrm{in}^{2}$. Unlike the deformation which for a given $\mathrm{x}$, $\mathrm{y}$ point is the same at all $\mathrm{z}$ distances into the crystal, the components of the stress tensor change sign with $\mathrm{z}$ distance into the crystal. Thus it is best to examine the $\Delta \mathrm{n}=\mathrm{n}_{\mathrm{e}}-\mathrm{n}_{\mathrm{o}}$ distribution at a single z-location in the crystal rather than analyze the distribution for the full volume at once. Figure 5 shows a histogram of the calculated $\Delta \mathrm{n}(\mathrm{x}, \mathrm{y})$ at the top surface of the crystal. The histogram shows that essentially all of the values lie between $2 \times 10^{-7}$ and $4 \times 10^{-7}$. These values can be equated to an equivalent detuning angle. One assumes that the $\Delta \mathrm{n}$ is due to a local rotation of the crystal z-axis.

$$
\Delta \mathrm{n}=\partial \mathrm{n}_{\mathrm{e}}(2 \omega) / \partial \theta \times \Delta \theta
$$

For a Type I doubler, $\partial \mathrm{n}_{\mathrm{e}} / \partial \theta=4.2 \times 10^{-8} / \mu \mathrm{rad}$ so the detuning angle range corresponding to the $\Delta \mathrm{n}$ range in the histogram is from $-4.8 \mu \mathrm{rad}$ to $9.4 \mu \mathrm{rad}$. However as can be seen from the histogram most of the $\Delta \mathrm{n}$ distribution is between $\pm 2 \mu \mathrm{rad}$. From the above analysis on the effect of the detuning angles due to deformation, it can be concluded that the stress-optic effect has an smaller effect on $3 \omega$ conversion efficiency that the deformation.

\section{CONCLUSION}

We have modeled the effect of gravity on the deformation and stress of a horizontally oriented KDP Type I doubler. The results have shown that gravity sag has a small effect on tripling efficiency when the crystal is supported fully around its periphery. A four point mounting of the crystal however would result in large decrease in conversion efficiency.

\section{REFERENCES}

1. Landolt-Bornstein, Numerical Data and Functional Relationships in Science and Technology, Volume 11, Springer-Verlag, New York 1984.

2. Maker, B.N. "NIKE3D, A Nonlinear, Implicit, Three-Dimensional Finite Element Code for Solid and Structural Mechanics - User's Manual”, Lawrence Livermore National Laboratory Report UCRL-MA-205268 Rev. 1, April 14, 1995.

3. Nye, J.F., Physical Properties of Crystals, Their representation by tesnors and matrices, Oxford University Press, London 1986.

4. Narasimhamurty, T.S. , Photoelastic and Electro-Optic Properties of Crystals, Plenum Press, New York 1981. 


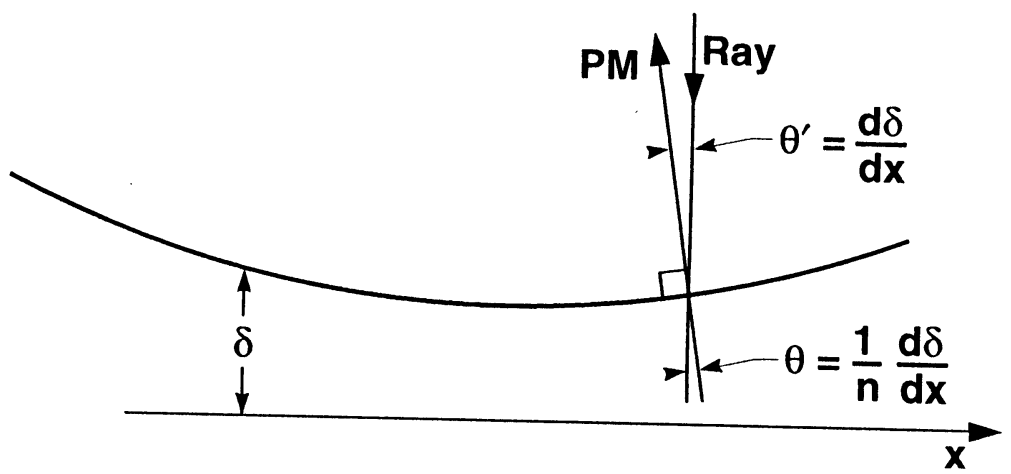

1. Deformation of the crystal results in a detuning from the phase-matching condition.

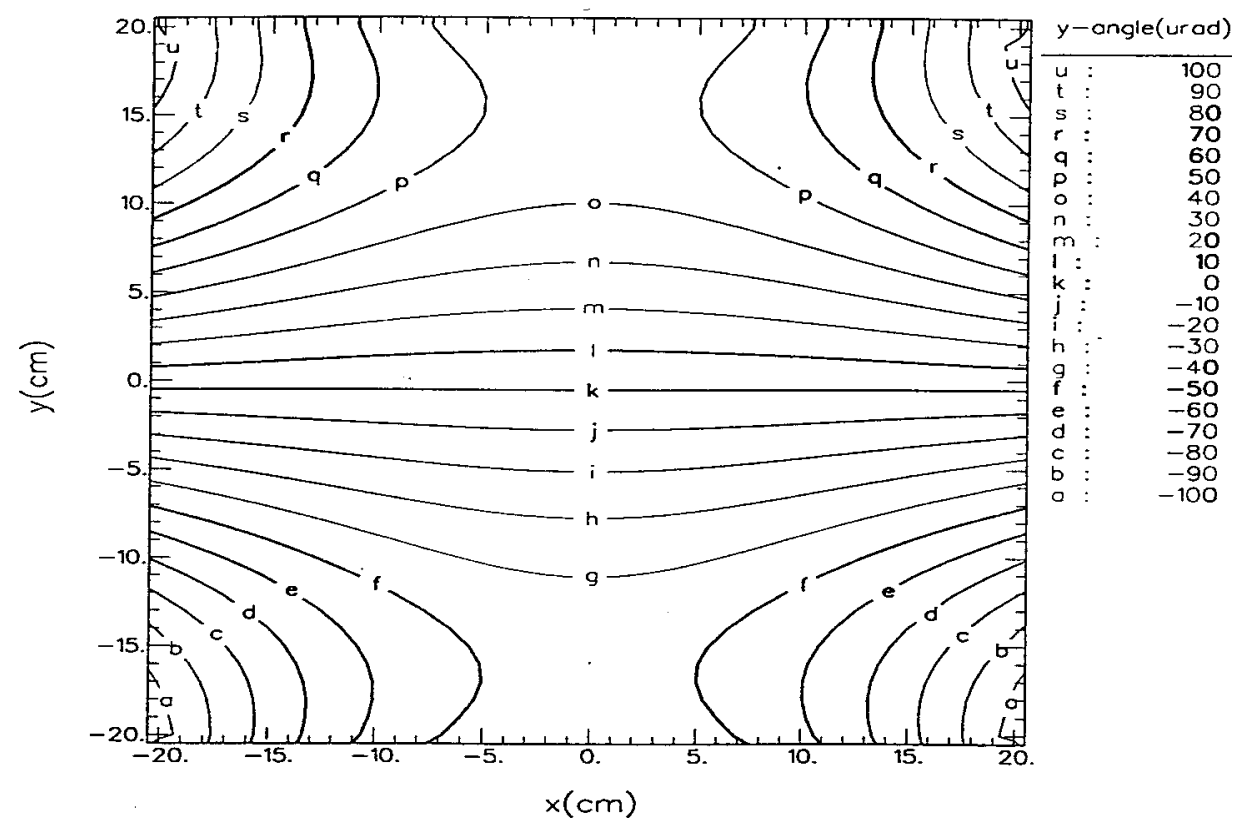

2. Detuning angle distribution for the doubler crystal supported at 4 corners. 


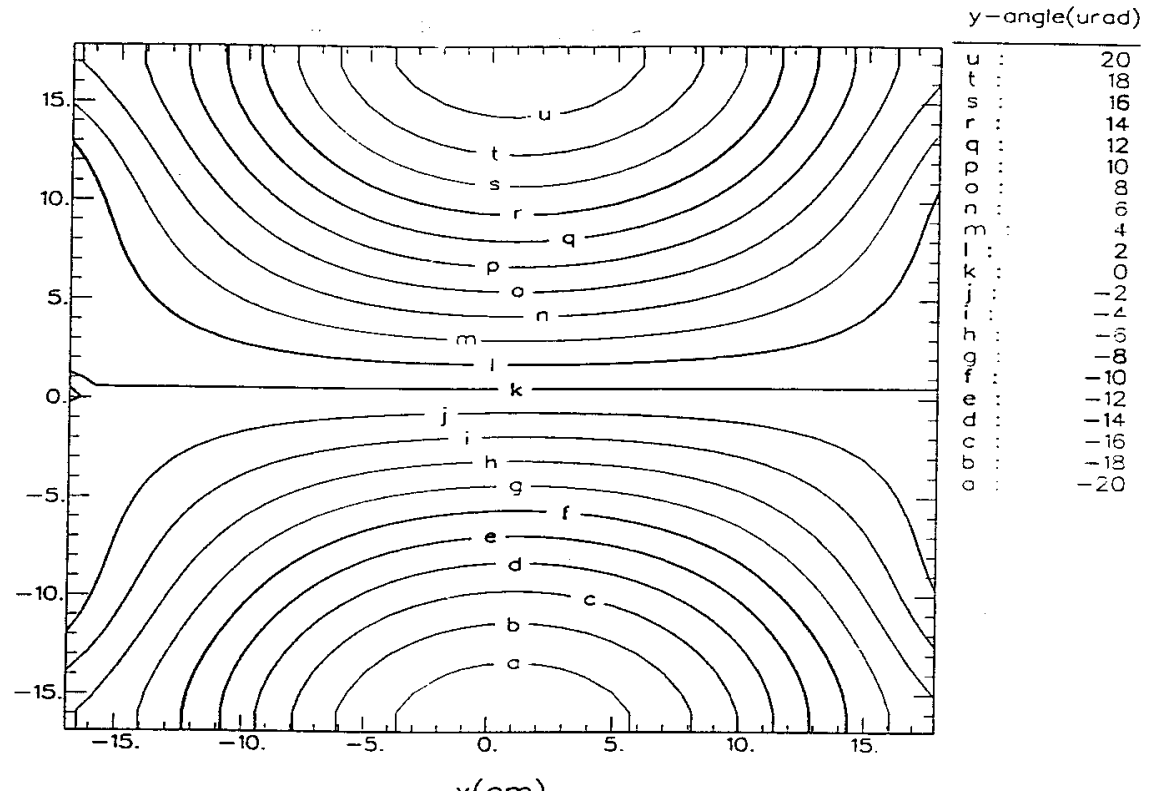

3. Detuning angle distribution for the full periphery simply supported doubler crystal.

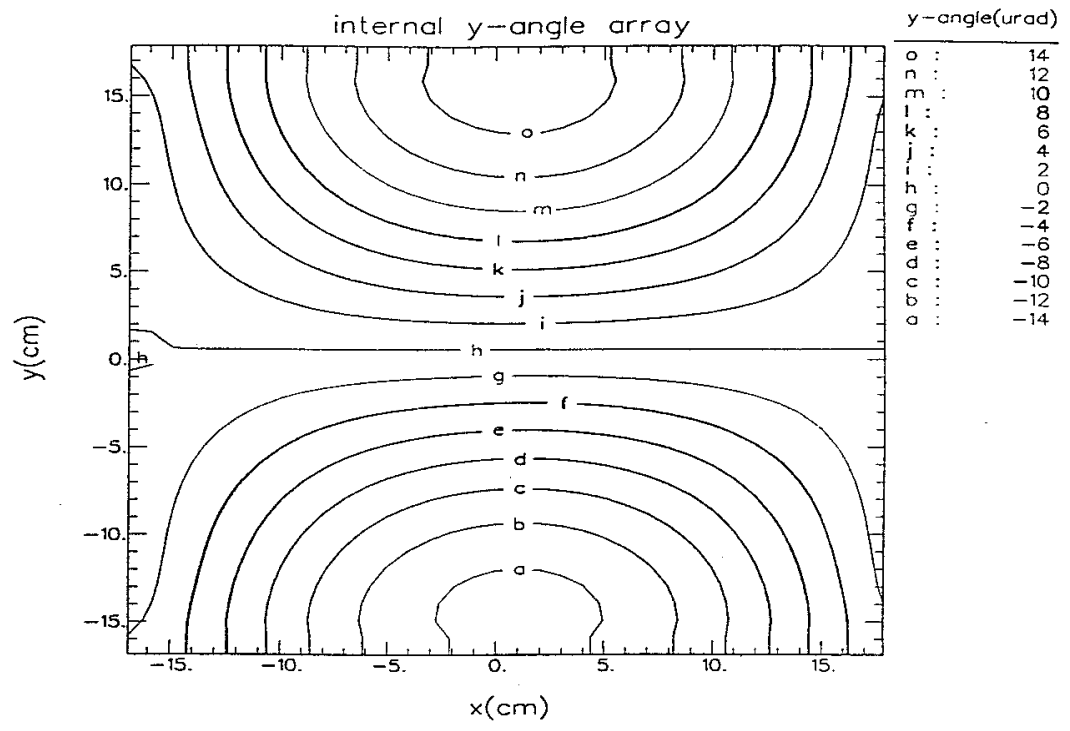

4. Detuning angle distribution for the full periphery clamped doubler crystal. 


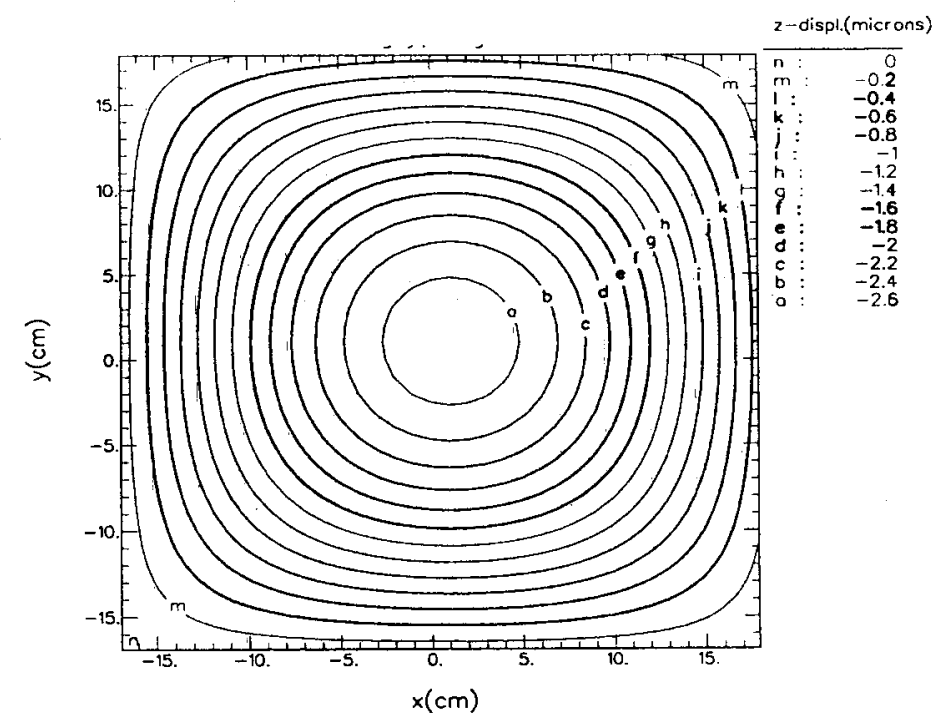

5. Z-displacment distribution for the clamped doubler crystal.

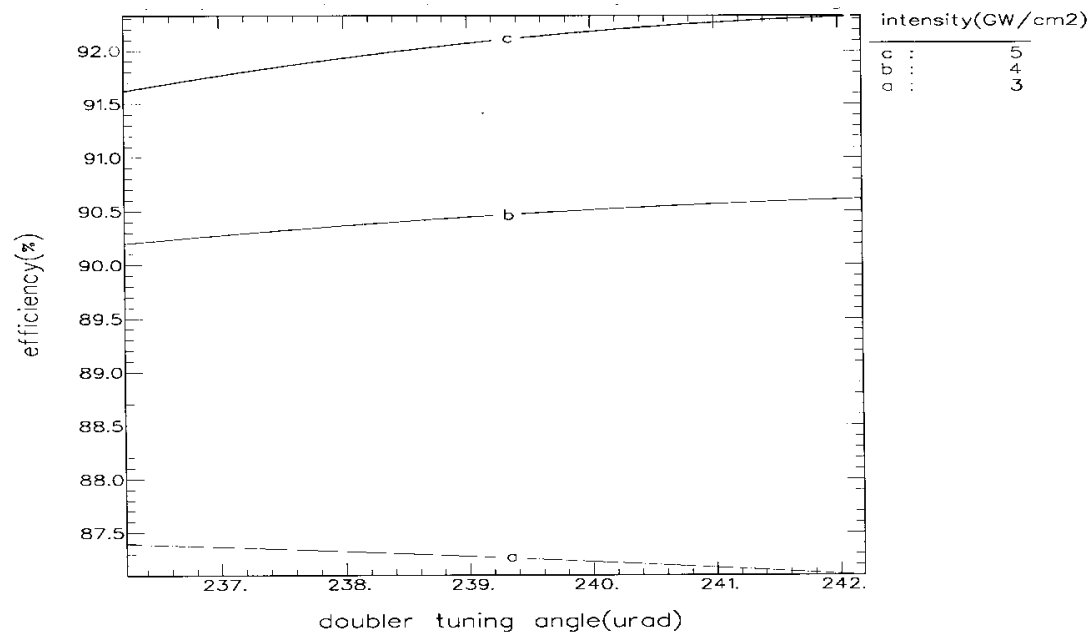

6. Tripling efficiency over the range of sag angles for the clamped doubler case for 3 different $1 \omega$ input intensities. 


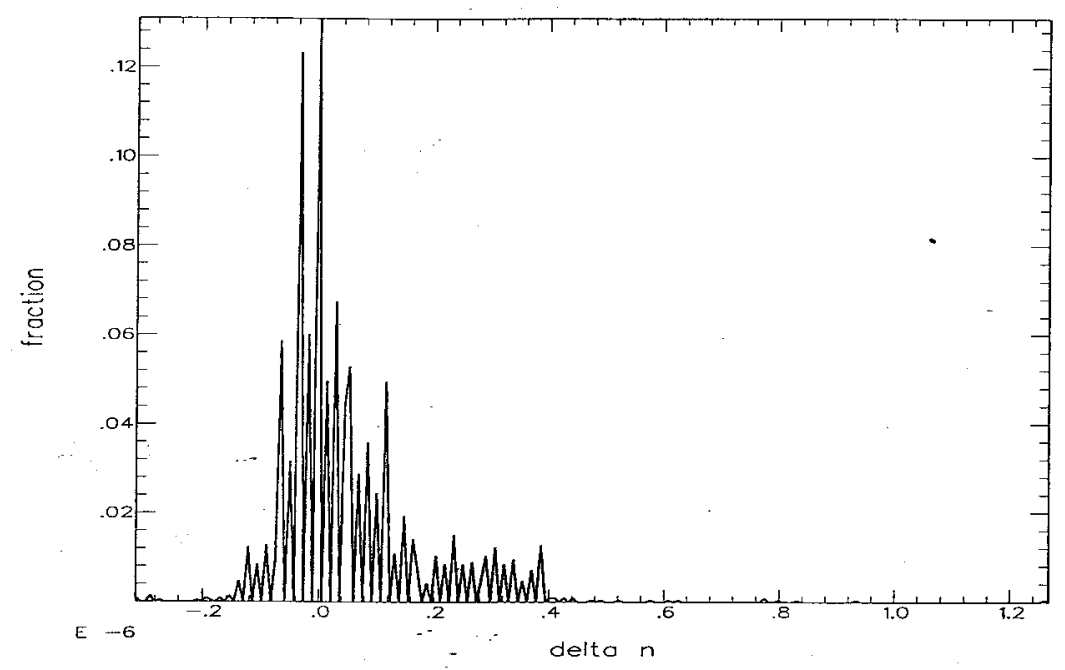

7. Histogram of the $\Delta \mathrm{n}$ values due to stress-optic effects in the clamped doubler 